Instituto Internacional de Investigación y Desarrollo Tecnológico Educativo INDTEC, C.A.

DOI: https://doi.org/10.29394/Scientific.issn.2542-2987.2020.5.15.4.87-105

OAI-PMH: http://www.indteca.com/ojs/index.php/Revista Scientific/oai

Artículo Original / Original Article

\title{
Hacia una Teoría del Riesgo Humanístico en la Responsabilidad Social de Garantías Recíprocas desde la Complejidad
}

\author{
Autor: Carlos Liborio Camacho Quintero \\ Universidad de Los Andes, ULA \\ ccamacho@ula.ve; clcamachoq71@gmail.com \\ Mérida, Venezuela \\ https://orcid.org/0000-0002-7552-5245
}

\section{Resumen}

El objetivo del artículo es generar una teoría humanista basada en la responsabilidad social, con la finalidad de indagar las garantías reciprocas en tiempos complejos, ontológicamente el ser y el hacer como proceso integral, examinando el efecto del otorgamiento de la fianza sobre el riesgo de la sociedad. Actualmente el sistema nacional de garantías muestra principios axiológicos en la cultura de riesgo del ser humano. En las instituciones financieras la ambigüedad y entropía genera baja liquidez exponiendo altos niveles de incertidumbre lo que hace que el apalancamiento sea ineficiente. Metodológicamente se empleará el paradigma cualitativo de tipo fenomenológico, tomando en consideración a los informantes clave el cual estará conformado por cuatro (04) gerentes o propietarios de Pequeñas $Y$ Medianas Empresas (PYMES), ubicadas en el Municipio Libertador y Campo Elías. Este ambiente inestable hace al sistema financiero sensible a la hiperinflación por los altos costos ya que no existe una competencia perfecta en la economía, asimismo, el exiguo crecimiento cambiario por su complejidad es detonante para las instituciones en las cuales afecta tanto micro como macroeconómicamente la ventaja competitiva sustentable y sostenible.

Palabras clave: responsabilidad social; humanismo; análisis económico; globalización.

Cómo citar este artículo:

Camacho, C. (2020). Hacia una Teoría del Riesgo Humanístico en la Responsabilidad Social de Garantías Recíprocas desde la Complejidad. Revista Scientific, 5(15), 87-105, e-ISSN: 2542-2987. Recuperado de: $\underline{\text { https://doi.org/10.29394/Scientific.issn.2542-2987.2020.5.15.4.87-105 }}$

Fecha de Recepción: 15-07-2019
Fecha de Aceptación:

25-11-2019
Fecha de Publicación: 05-02-2020 


\title{
Towards a Theory of Humanistic Risk in Social Responsibility of Reciprocal Guarantees from Complexity
}

\begin{abstract}
The objective of the article is to generate a humanistic theory based on social responsibility, with the purpose of investigating reciprocal guarantees in complex times, ontologically being and doing as an integral process, examining the effect of granting the bond on the risk of society. Currently, the national guarantee system shows axiological principles in the risk culture of human beings. In financial institutions ambiguity and entropy generates low liquidity exposing high levels of uncertainty which makes leverage inefficient. Methodologically, the phenomenological qualitative paradigm will be used, taking into account the key informants which will be made up of four (04) managers or owners of Small and Medium Enterprises (SMEs), located in the Municipality of Libertador and Campo Elías. This unstable environment makes the financial system sensitive to hyperinflation because of the high costs since there is no perfect competition in the economy, also, the low exchange rate growth due to its complexity is detonating for the institutions in which both the micro and macroeconomically affects the advantage competitive sustainable and sustainable.
\end{abstract}

Keywords: social responsibility; humanism; economic analysis; globalization.

How to cite this article:

Camacho, C. (2020). Towards a Theory of Humanistic Risk in Social Responsibility of Reciprocal Guarantees from Complexity. Revista Scientific, 5(15), 87-105, e-ISSN: 2542-2987. Recovered from: https://doi.org/10.29394/Scientific.issn.2542-2987.2020.5.15.4.87-105

Date Received:

15-07-2019
Date Acceptance:

25-11-2019
Date Publication: 05-02-2020 


\section{Introducción}

La génesis empresarial en los países globalizados está compuesta por Pequeña y Medianas Empresas (PYMES), las cuales son de gran importancia a la economía mundial, incrementando la sinergia entre el desarrollo empresarial y crecimiento económico, sin embargo, las empresas presentan fortalezas y debilidades en sus recursos financieros ya que los proyectos nuevos generan incertidumbre y riesgo por lo volátil del mercado, lo que influye en el valor de la garantía en los créditos correspondientes a las políticas para que el otorgamiento que visualizan los estados financieros de los préstamos avalados por los bancos.

Es importante mencionar que el sistema como estrategia asociativa entre grandes pequeñas y medianas empresas aporta al ciudadano común una iniciativa que apoya la creación y crecimiento, así como favoreciéndolas en el principio de sostenibilidad en el tiempo, generación de empleos y acceso a las mejores condiciones de financiamiento, donde los bancos y instituciones financieras deben cumplir las auditorias en las aristas de inspección, regulación, supervisión, control y vigilancia, igualmente estas instituciones son de mayor trascendencia por la función que desarrolla en beneficio de la pequeña y mediana empresa; en cuanto al otorgamiento de garantía para avalar el crédito en las sociedades de garantías.

Al respecto, la Constitución de la República Bolivariana de Venezuela (1999), en su artículo 308, establece que: "el Estado protegerá y promoverá la pequeña y mediana industria, las cooperativas, las cajas de ahorro, así como también la empresa familiar, la microempresa y cualquier otra forma de asociación comunitaria para el trabajo" (pág. 151); "con el fin de fortalecer el desarrollo económico del país (...)" (pág. 151).

Esbozando el artículo anterior, considera que este sistema es facilitador para realizar coyuntura, alianza e inclusión de pequeños y medianos empresarios al sistema financiero, sin embargo, el crecimiento del producto 
interno bruto (PIB) nos indica si estas pequeñas y medianas empresas (PYMES) son generadoras de empleo y progreso en la calidad de vida de la población.

En este sentido, Chiavenato (2006): define "cambio significa pasar de un estado a otro. Es la transición de una situación a otra. El cambio implica transformación, perturbación, interrupción y ruptura, que dependen de su intensidad (...) (pág. 150). Asimismo, el Sistema Nacional de Garantías Recíprocas, atiende a todos los sectores productivos del estado, destacándose hasta ahora el mayor repunte en el sector comercio y servicios respectivamente. Por lo tanto, las PYMES como agentes sociales tienen como finalidad robustecer la formación y capacitación de los ciudadanos, es decir, el desarrollo social afianzado en las finanzas para la unificación en la política y sociedad como impulso para el progreso del cumplimiento normativo.

De tal manera, el riesgo humanista es complejo en lo individual y colectivo en las instituciones comprometidas para la gestión en procesos de cambio, donde las tecnologías de información y comunicación están inmersas en la globalización, como necesidad para transfigurar el contexto humana, como reto en abordar y motivaren todo lo que nos encierra. Al respecto, Rojas (2018), expone:

Ahora bien, la responsabilidad social en la universidad en tiempos de posmodernidad genera nociones, generalidades que pueden ocasionar críticas o diatribas, si enfocamos la universidad desde segmentos dirigidos a la consecución de propósitos en mejora de los contextos sociales que incumben a su organización educativa. Asimismo, a la universidad, se le confiere el título de crear ideas, sustentadas en el cumplimiento de roles actuales planteados como es de esperar (pág. 310).

Por lo que la complejidad desde el sistema nacional de garantías constituye procesos de otorgamiento en avales, pero constituyen parte muy importante en la discusión del progreso económico de los emprendedores a 
nivel nacional. Sobre esta inferencia se pretende establecer nociones con intenciones de aportar explicaciones que den cuenta del estado actual de las mismas, así como hacer explícitas sus relaciones con la teoría vigente.

Por ello, el argumento investigativo en la sociedad de garantías reciprocas del Estado Mérida, describen la necesidad de compartir riegos, diversificar y optimizar los recursos de la institución a un costo fiscalizado, como secuela el análisis del riesgo tiene que ver con las garantías reales, generando como causas la ausencia de proyectos factibles ocasionando malestar financiero en los funcionarios, igualmente es necesario para estos estudios financieros realizar diagnósticos preventivos a través de la asistencia técnica.

El mercado financiero considera la eficiencia de estos proyectos, porque desarrolla las capacidades en la sociedad de garantías en la evaluación del riesgo del portafolio de créditos avalados por la sociedad, el comportamiento de las instituciones financieras trasladan los créditos hacia aspirantes con mejor calidad de garantías, cuya finalidad es evitar inconvenientes en las PYMES y aminorar los riesgos existentes para mejorar la calidad de vida en condiciones inmejorables como fuente principal de empleo.

En este momento, los distintos modelos de Sistema de Garantías coexisten y conviven con distintos procedimientos financieros. Emergiendo para cada caso una experiencia diferente de acuerdo a los motivos, etapas históricas que impulsaron su adopción en el principio particular de cada época, asimismo, las PYMES aportan al sector público en toda su estructura como piedra angular y columna vertebral para el fortalecimiento de estos enseres que sirva de apoyo a la responsabilidad social.

Los modelos de sistema de garantías en sus capítulos II y III esgrime en las disposiciones que regula el Sistema Nacional de Garantías Reciprocas enfocado en los decretos de rango y fuerza de ley, se atisba fundamentalmente el financiamiento directo iniciando el reafianzamiento, es 
decir, comprimir el desgaste de la morosidad ya que las sociedades tienen como objetivo la integración de las sociedades para que se pueda desarrollar el coafianzamiento productivo.

Los fondos respaldan los ordenamientos en las sociedades de garantías patrimoniales con el sector económico en la capital del País, suministrando proyectos y programas de crédito que consoliden la normativa, procedimientos y reglamentos del ejecutivo de bancos e instituciones financieras, asimismo, los fondos de garantías se transforman en sociedades anónimas para el sector público de las PYMES.

Las instituciones de garantías establecen sus fianzas con el reembolso de créditos concedidos a los accionistas públicos o privados, que están ajustados en la ley de bancos, tanto para el ahorro o préstamo, así como también, sin embargo los socios pueden participar en subastas y capacitar mediante asesoramiento técnica y financiera en la gestión económica, del mismo modo, las sociedades de garantías conceden saneamientos tanto en la actividad económica como multisectoriales, para reforzar las actividades financieras, por lo tanto, tienen como finalidad, consolidar fianzas, reembolsos y créditos en licitaciones.

En Venezuela, según la Ley del Instituto Nacional de Desarrollo de la Pequeña y Mediana Industria (INAPYMI, 2014), en su Artículo 5, se expresa que a los efectos del presente Decreto con Rango, Valor y Fuerza de Ley se entiende por Pequeña y mediana Industria: "toda unidad organizada jurídicamente, con la finalidad de desarrollar un modelo económico productivo mediante actividades de transformación de materia prima en insumos, en bienes industriales elaborados o semielaborados, dirigidos a satisfacer las necesidades de la comunidad" (pág. 12).

El índice de solvencia financiero en la responsabilidad social de los riesgos pertenece a la sociedad de patrimonio y saldos exigibles por las garantías en cada trimestre que como requisito es la vigencia, debe analizarse 
con el Ejecutivo Nacional en las sociedades tributarias que ocasione la norma, de igual manera se debe considerar la incertidumbre al momento de otorgar una garantía ante el riesgo de liquidez en la crisis bancaria, otro factor es minimizar el riesgo de default a los beneficiarios que no cancelen su deuda y el riesgo de mercado que es de gran importancia macroeconómica en el portafolio bancario y financiero.

En cuanto a los riesgos de liquidez, este se reduce cuando el sistema de garantías es institucional, es decir, los bancos establecen políticas para la venta de créditos y generar un mercado acorde al financiamiento, pero trae sus fortalezas y debilidades en el cual existe un menor riesgo de liquidez, las PYMES incrementa tasa de financiamiento y extienden los plazos de su cancelación, ahora bien, el fenómeno riesgo asociado a la cartera de beneficiario de la Sociedad de Garantías Reciprocas del Estado Mérida, se encuentra conformada por fianzas financieras, la finalidad es garantizar a través de licitación el cumplimiento técnico o profesional la obligación adquirida y así quede avalada por un tercero.

El sistema de reafianzamiento de operaciones permiten diversificar el riesgo y optimizar los recursos de las sociedades de garantías, los socios participan en el pago del crédito, de no realizarlo se procede a demandarlo brindando al conjunto del sistema de garantías, un mayor nivel de liquidez y expansión, por lo tanto el precio es factor importante en la garantías, los riesgos siempre están presentes, es una entropía que ocurre en los bancos, cuya finalidad es minimizar el desorden con respecto al riesgo por la entidad de financiamiento llevando a una inexorable valoración, control y seguimiento en las operaciones, asimismo, el incremento en las garantías no penaliza ni castiga el mal funcionamiento en la eficacia del portafolio el cual va a garantizar un mejor desempeño bancario.

Existen varios factores que coadyuvan al crecimiento del riesgo si no son controlados, entre ellos se tiene el que se encuentra politizado, donde el 
financiamiento en la PYMES es factor de estudio para el gobierno, porque la obligación facilita el crédito en cada solicitud en los países que solicitan este aval de financiar, las políticas públicas aunado con los derechos humanos son factores de garantías ante un riesgo político, sin embargo la política mal orientada y sin liderazgo no favorece los consumidores ante el gobierno que este dirigiendo un país, asimismo, la supervisión debe estar regulada y normada para evitar riesgos financieros, es necesario diseñar programas en los bancos con respecto a los interés que no sean tan altos que destruyan al sector público.

Al cabo, los administradores de bancos deben planificar y organizar un mejor control del precio en la garantía para que sean capaces de la sostenibilidad en los costos de operación, por lo tanto, las PYMES no deben verse como débiles al sistema financiero más bien que sirvan de apoyo. De igual manera, si el precio de la garantía es muy elevado se requiere hacer uso de la técnica del reafianzamiento como medida para diversificar el riesgo cosa que la Sociedad de Garantías Reciprocas del Estado Mérida presenta debilidad.

Sin embargo, la seguridad jurídica utiliza reglamentos los cuales están normados en contratos y su cumplimiento es formal, las empresas deben estar en la vanguardia en generar estrategias para que los créditos y garantías sean asequibles para los beneficiarios y así buscar que los bancos amplíen su portafolio para nuevos inversionistas en bienes muebles e inmuebles, así como una adecua capitalización a las sociedades de garantías reciprocas.

Se puede destacar, que en el caso venezolano, específicamente la sociedad financiera de garantías reciprocas del estado Mérida, experimentan criterios prudenciales para el manejo de riesgo como son la constitución de provisiones, el reafianzamiento de las operaciones, el cofinanciamiento, el riesgo y la constitución patrimonial humanístico en la sociedad, prácticas que se han venido desarrollando muy poco y que deben mejorar con la finalidad 
de mitigar y controlar el nivel de endeudamiento.

El presente artículo, busca generar una teoría humanística del riesgo financiero, por lo que vale indagar ¿Cómo vive la experiencia del riesgo de la responsabilidad social de garantías reciprocas en el Estado Bolivariano de Mérida?, por lo que esta interrogante admite formular las siguientes intencionalidades: Explorar el fenómeno del riesgo financiero, develar los elementos subyacentes en el fenómeno del riesgo y resignificar los hallazgos teóricos en el fenómeno humanista del riesgo financiero en la sociedad de garantías recíprocas del estado Bolivariano de Mérida.

\section{Contexto Teórico}

Se analizan diversas teorías para lo cual se aborda inicialmente el paradigma humanista y complejidad, igualmente la sociedad de garantías reciprocas y el riesgo financiero, lo que permite tener un fundamento doctrinario en la investigación. La complejidad rompe paradigmas, su pensamiento sistémico, hologramático y dialógico se enfocan en la teoría de caos, es decir, del desorden al orden uno extirpa al otro, la gestión tecnológica de información y comunicación de las PYMES hacia la perspectiva de la gerencia interdisciplinaria del Siglo XXI, es un proceso que en las últimas décadas ha sufrido cambios en todos los ámbitos: políticos, económicos, sociales, culturales, ambientales, como consecuencia de la globalización y Venezuela no escapa de ella.

Cabe destacar entre estos cambios, las nuevas tecnologías de información y comunicación TIC, desde donde vale la pena preguntarse si realmente han brindado soluciones a países en vías de desarrollo, que hasta hoy presentan graves problemas derivados principalmente por la falta de productividad y diversificación del aparato productivo nacional, por lo que, las PYMES no escapa de estas dificultades, ya que estas desempeñan un papel protagónico como responsables en fortalecer el crecimiento hacia el desarrollo 
económico de los países, las cuales organizan parte de la estructura empresarial a nivel global; debido a su aporte para la generación del ingreso nacional, diversificación del aparato productivo, además de la creación de nuevas fuentes de empleo.

En tal sentido, las PYMES fortalecen la economía de cualquier país, generando diversificación del aparato productivo y produciendo nuevas fuentes de empleo, además de la incorporación de nuevas tecnologías en el área de la productividad aunada al apoyo del Estado en el otorgamiento de financiamiento y la formación permanente de los gerentes. En este mismo orden de ideas, considerando la fortaleza, capacidad e interés dentro de cada una de ellas, permite una perspectiva interdisciplinaria que demanda la gerencia moderna, asimismo, condujo a que el ser pensante intentara humanizar a las ciencias mediante las relaciones humanas, destacando la importancia del individuo, especialmente, el libre albedrío, la creatividad y la espontaneidad; esto quiere decir, que el protagonista de todos los procesos gerenciales de las organizaciones es el ser humano, atribuyendo a lo positivo, en la satisfacción de las relaciones laborales, lo que conlleva hacia la gerencia interdisciplinaria.

\section{Metodología}

Para establecer los criterios epistemológicos metodológicos, se hace uso como compendios el paradigma cualitativo a través de la metodología fenomenológica como atisbo filosófico sobre la percepción de los hechos entre el fenómeno estudiado y el supuesto que indica cómo se percibe las experiencias, los informantes clave estará conformado por cuatro (04) gerentes o propietarios de PYMES ubicadas en el Municipio Libertador y Campo Elías, donde se establecieron como características principales: sector productivo, tiempo de la actividad productiva. Se considera mayor a 15 años, números de trabadores, operatividad de la unidad productiva, tipo de actividad 
productiva, comercialización de los productos y ubicación geográfica; como técnica se utiliza la entrevista en profundidad con la observación participante la cual proyecta resultados en las subcategorías que emergen de estos informantes de acuerdo al proceso de análisis del riesgo financiero permitiendo orientar el estudio hacia una teórica humanística del riesgo en el sistema nacional.

En la tabla 1, se presentan los gerentes de las PYMES seleccionadas para la presente investigación donde se destaca que las mismas, están ubicadas en dos (02) Municipios del Estado Mérida, Libertador (PyMIL1, PyMIL2) y Campo Elías (PyMIA1, PyMIA2).

Tabla 1. PYMES seleccionadas para el estudio.

\begin{tabular}{|c|c|c|c|}
\hline No $^{\circ}$ & Identificación & $\begin{array}{c}\text { Ubicación Geográfica } \\
\text { Estado Mérida }\end{array}$ & Características Productivas \\
\hline 1 & Ge-PyMIL1 & Municipio Libertador & $\begin{array}{c}\text { Procesamiento de productos textil } \\
\text { (carteras y bolsos para dama) }\end{array}$ \\
\hline 2 & Ge-PyMIL2 & Municipio Libertador & $\begin{array}{c}\text { Procesamiento de productos de } \\
\text { limpieza personal (jabones) }\end{array}$ \\
\hline 3 & Ge-PyMIA1 & Municipio Campo Elías & $\begin{array}{c}\text { Procesamiento de productos } \\
\text { agroindustriales (pulpa de frutas) }\end{array}$ \\
\hline 4 & Ge-PyMIA2 & Municipio Campo Elías & $\begin{array}{c}\text { Procesamiento de materiales de } \\
\text { construcción (Fabrica de bloques } \\
\text { de cemento) }\end{array}$ \\
\hline
\end{tabular}

Fuente: El Autor (2019).

La información alcanzada, se organiza en categorías, subcategorías, dimensiones y se analiza a través de la triangulación para discrepar las opiniones documentadas, utilizando la etnografía; con el fin de contextualizar la realidad existente con la teoría, asimismo, el estudio se apoya en teorías con perspectivas tanto humanistas como del pensamiento complejo examinando las acciones, necesidades básicas y comportamientos de los informantes clave en su campo de acción, de acuerdo a la función social de 
cada uno de ellos.

Del mismo modo, Heidegger (2005), citado por Barbera e Inciarte (2012), expresan que:

Para quien su principal tarea fue enseñar el arte de pensar. Tradicionalmente se entiende el pensar como un simple relacionar, es decir, poner una cosa en determinada relación y sobre la base de esta relación se hace una afirmación que es denominada juicio (pág. 202).

Esgrimiendo a estos autores, los aportes que se pueden realizar desde esta pluridimensionalidad son de gran importancia para el ser humano y para la sociedad. Se explica entonces que, el enfoque o paradigma interpretativo va al encuentro de razones y no de causas como en las visiones clásicas, sin embargo, la fenomenología asumida como paradigma interpretativo con perspectivas teóricas genera la comprensión de significados en los fenómenos de los informantes clave.

Siguiendo estas ideas, Martínez (2007), expone que:

El método fenomenológico se enfoca en analizar las experiencias y vivencias que no son del todo comunicables, sino que más bien subyacen a las acciones realizadas por el fenómeno, donde se observa que los resultados incuestionables en el análisis exhaustivo para la interpretación de las subcategorías que emergen de cada actor social en la efectividad de la búsqueda de asirse en las experiencias vividas (pág. 139).

Ahora pues esas experiencias vividas en el ser axiológico, indaga sobre la naturaleza de la realidad que circunda alrededor de los distintos trabajadores de la Sociedad de Garantías, de lo que existe, del ser y cómo éstos perciben su entorno. De acuerdo con Castro y Guzmán (2005): "los estilos de aprendizaje señalan la manera en que el estudiante percibe y procesa la información para construir su propio aprendizaje, éstos ofrecen indicadores que guían la forma de interactuar con la realidad" (pág. 87). 
Posteriormente, dentro del entorno investigativo permitirán emerger subcategorías en la entrevista en profundidad del material seleccionado como las grabaciones y notas de campo, que serán las fases en la unidad de análisis, categorización, estructuración, triangulación y teorización, en consecuencia, se genera una teoría humana desde el entorno de la complejidad que explique el fenómeno que es producto de la experiencia vivencial de la información recabada acerca de estos actores sociales.

\section{Análisis de los Hallazgos}

En la información analizada de los informantes clave de las categorías derivadas de la misma, generaron el proceso de categorización en el cual emergió como la más resaltante la subcategoría conformismo como detonante de la insostenibilidad financiera de la sociedad de garantía reciprocas, luego surge la categoría bien conocida en estos ámbitos como el amiguismo aspecto importante relacionado con lo mejor conocido como el favoritismo que no es un tema nuevo en el mundo laboral y que en este caso según resultados de los entrevistados, tres de ellos manifestaron haber llegado a la sociedad de garantía a través de un amigo o por ser amiga de la presidenta de la institución, a continuación se menciona en la tabla 2, las subcategorías y meta-categorías en los gerentes de las PYMES.

Tabla 2. Subcategorías y meta-categorías en los Gerentes de las PYMES.

\begin{tabular}{|c|c|c|}
\hline Subcategoría & $\begin{array}{l}\text { Conformismo } \\
\text { Detonante }\end{array}$ & $\begin{array}{l}\text { - Desconocimiento de Manuales. } \\
\text { - Inexistencia de Indicadores. } \\
\text { - Inexistencia de un Sistema Riesgo. } \\
\text { - No existe compromiso institucional. }\end{array}$ \\
\hline \multirow{5}{*}{$\begin{array}{c}\text { Meta- } \\
\text { categorías }\end{array}$} & \multicolumn{2}{|c|}{ - Insostenibilidad Financiera de la SGR, Mérida. } \\
\hline & \multicolumn{2}{|c|}{ - Nuevo Servidor Público. } \\
\hline & \multicolumn{2}{|c|}{ - Decisiones Centralizadas. } \\
\hline & \multicolumn{2}{|c|}{ - Deficiencia Control y Seguimiento. } \\
\hline & \multicolumn{2}{|c|}{ - Patrimonio Devaluado. } \\
\hline
\end{tabular}

Fuente: El Autor (2019). 
Ahora bien, esbozando la tabla 2, la subcategoría conformismo es el detonante más contundente en cuanto a los hallazgos, la cual se toma como el área temática central del presente artículo, al considerarla el detonante principal de la investigación, igualmente, se observa una mirada encontrándose con un patrimonio devaluado, al cual no se le ha hecho seguimiento por parte de sus accionistas y entes reguladores, trayendo como consecuencia la generación de bajos ingresos para cubrir gastos.

Luego se valoraron aspectos como son la no existencia de indicadores, sistemas de riesgo, compromiso institucional, el personal desconoce la existencia de los manuales de normas y procedimientos y volvió a surgir la interrogante ¿cómo es que el personal se siente cómodo? Y se me viene a la mente que como los gastos por sueldos y salarios para todo el personal de la sociedad provienen y están garantizados por el gobierno nacional, donde las PYMES del Municipio Libertador y Campo Elías buscan incrementar el número de operaciones en otorgamiento de fianzas y que baje o suba el nivel de riesgo, la finalidad es mejorar los beneficios de ley entre ellos un bono de productividad establecido por el Gerente y que debe ser cancelado por cumplimiento de meta, y como estos recursos están garantizados ellos no tienen por qué preocuparse y mientras ellos se encuentren en su zona de confort no pensarían en ningún cambio.

\section{Propuesta}

La propuesta está enfocada en el Conformismo como el detonante para la insostenibilidad financiera de la sociedad de garantías reciprocas, es el hallazgo más importante que permite mejorar las condiciones al empleado público que se encuentra sumergido en el conformismo y desmotivación, como un ser óntico capaz de perturbar el conjunto de relaciones que se establecen en su entorno laboral cuya teoría humanista va orientada en mejorar su status quo y felicidad. Indico las aristas más importantes de la teoría humanista desde 
la complejidad.

Zona de aprendizaje: esta actitud reflejada en la comodidad de los trabajadores también se encuentra afectada por el comportamiento de los supervisores al momento de tomar decisiones, el subordinado es afectado, igualmente, la falta de capacitación perturba su vida laboral es donde el nivel gerencial, es un factor de dialogo y sinergia holística, ocasionando estancamiento en el personal, desmotivación, sin embargo, esto da apertura a nuevos retos porque son considerados en momentos y decisiones importantes.

Sostenibilidad financiera: es necesario que la gestión sostenible debe servir de columna vertebral para cualquier duda que se tenga al respecto. La gerencia el líder financiero es corresponsal en las expectativas de los inversores, el compromiso está orientado en la sostenibilidad para que la alta dirección asuma riesgos financieros en sus garantías y valores, la cual debe transformarse en base sólida, para que la sinergia laboral se convierta en un proceso de diferenciación y distinción. Entre los síntomas de la insostenibilidad tenemos:

- Alto endeudamiento.

- Deficiencia organizacional, administrativa, financiera y empresarial.

- Problemas en la empresa.

- Baja rotación de cartera.

- Fenómenos naturales.

Sociedad y conciencia: dentro de la complejidad el principio de autonomía y dialógico hacen una imbricación entre sociedad y conciencia en la creación ontológica como protagonista, este ser debe ser capaz de trabajar en equipo en calidad de servicio, entrega y ejecución, donde la alteridad axiológica lo transforma en solidario, humano, respetuoso, altruista, filántropo 
y amistoso; capaz de ver en los demás trabajadores la capacidad armoniosa y colaboradora en la misión y visión de la empresa. Por tanto, la capacitación es importante, un ser humano cognitivo se prepara, aprende y se hace más sabio para un mejor servicio, exigiéndose a sí mismo la excelencia, soñador y prospectivo en la planificación de nuevos retos financieros.

Servidor Público: el objetivo fundamental es mejorar el modelo económico productivo en las PYMES donde el humanismo nos lleva a empoderarnos y marca la pauta en un trabajador sensato que pueda dar lo máximo dentro de la institución pública, es decir, un coach ontológico que busque mejorar los riesgos financieros. Esta es una proposición que va de la base a la punta de la pirámide horizontal de poder del gobierno y el estado, creando procesos de transformación, liderazgo participativo y autonomía en todos los niveles. Se observa en la figura 1, la construcción de la Teoría humanista basada en responsabilidad social del riesgo financiero en la sociedad de garantías recíprocas en tiempos complejos.

Figura 1. Teoría humanista basada en responsabilidad social del riesgo financiero en la sociedad de garantías recíprocas en tiempos complejos.

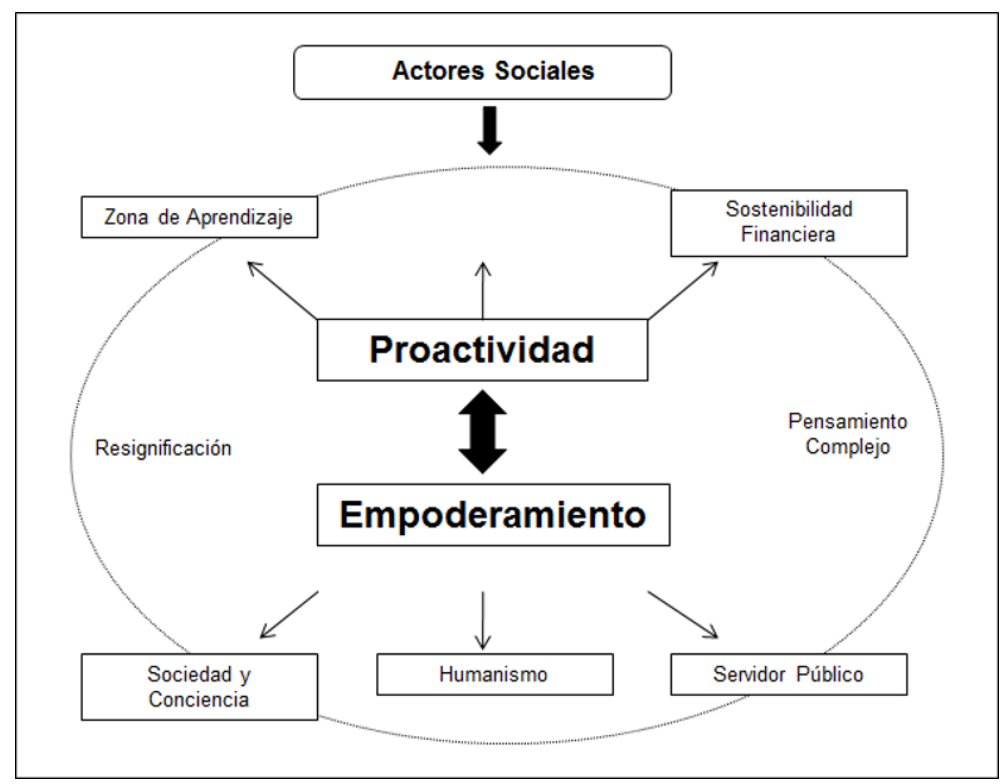

Fuente: El Autor (2019). 


\section{Reflexiones Concluyentes}

La investigación se efectuó desde la perspectiva fenomenológica, método vinculado con el paradigma cualitativo, y sobre la base de la complejidad y del humanismo, características propias de la postmodernidad, por lo que se puede concluir que luego de indagar el fenómeno del riesgo en la sociedad de garantías recíprocas, han develado los siguientes aristas generadas de las subcategorías emergentes de los informantes clave como son: administración pública, el amiguismo, el perfil del servidor, las decisiones centralizadas, desconocimiento de manuales y normas, el conformismo, toma de decisiones y patrimonio devaluado, dando paso a la resignificación.

Estas contribuciones permitan resignificar los objetivos planteados, unidad de análisis de las distintas categorías alcanzadas en el camino de la investigación, consistiendo en todo el proceso de categorización da paso a la teorización para darle significado a los términos derivados de la tipificación de las dimensiones recopiladas de los informantes clave, donde se atisba que el conformismo es el causal riesgo, la idea es a través del humanismo y complejidad empoderar al servidor público basado en principios y valores primordiales en la postmodernidad.

De esta manera, se propone un sujeto capaz de dar lo máximo de sí, evitar el conformismo como detonante de la insostenibilidad financieras, la idea es incrementar al trabajador en su crecimiento con consciencia para mejorar sus condiciones de vida. Así esto se traducirá en una sociedad de responsabilidad social financieramente para el desarrollo endógeno de la región, que conlleva a mejorar la calidad de vida y las relaciones productivas en las PYMES del Municipio Libertador y Campo Elías en el estado Mérida, es decir, que se pueda convertir en resignificación del gerente como patrón transformacional que minimice el conformismo. 


\section{Referencias}

Barbera, N., \& Inciarte, A. (2012). Fenomenología y hermenéutica: dos perspectivas para estudiar las ciencias sociales y humanas. Multiciencias, 12(2), 199-205. e-ISSN: 1317-2255. Recuperado de: http://produccioncientificaluz.org/index.php/multiciencias/article/view/1 $\underline{6900}$

Castro, S., \& Guzmán, B. (2005). Los Estilos de Aprendizaje en la Enseñanza y el Aprendizaje: Una propuesta para su Implementación. Revista de Investigación, (58), 83-102, e-ISSN: 07980329. Recuperado de:

https://www.redalyc.org/articulo.oa?id=376140372005

Constitución de la República Bolivariana de Venezuela (1999). Gaceta Oficial

Extraordinaria N. ${ }^{\circ} 36.860,30$ de diciembre. Caracas, Venezuela: Asamblea Nacional Constituyente.

Chiavenato, I. (2006). Introducción a la Teoría General de la Administración. Séptima Edición, ISBN 13: 978-970-10-5500-7. México: McGraw-Hill Interamericana.

INAPYMI (2014). Ley Para la Promoción y Desarrollo de la Pequeña y Mediana Industria y Unidades de Propiedad Social. Gaceta Oficial N. 6.151 Extraordinaria 18 de noviembre. Caracas, Venezuela: Instituto Nacional de Desarrollo de la Pequeña y Mediana Industria.

Martínez, M. (2007). Evaluación Cualitativa de Programas. México: Editorial Trillas.

Rojas, M. (2018). Nociones Críticas en la Responsabilidad Social de la Extensión Universitaria con la Vinculación Comunidad. Revista Scientific, 3(10), 304-316, e-ISSN: 2542-2987. Recuperado de: https://doi.org/10.29394/Scientific.issn.2542-2987.2018.3.10.17.304$\underline{316}$ 


\section{Carlos Liborio Camacho Quintero}

e-mail: ccamacho@ula.ve; clcamachoq71@gmail.com

Nacido en Mérida, estado Mérida, Venezuela, el 25 de

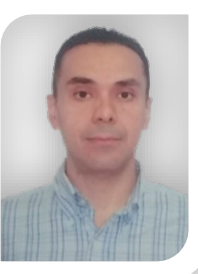
marzo del año 1971. Post-Doctor en Gerencia para el Desarrollo Humano (ULA); Doctor en Gerencia Avanzada; Doctorando en Ciencias de la Educación; Magíster Scientiarum en Gerencia Empresarial; Especialista en Telemática e Informática en Educación a distancia (EaD); Ingeniero de Sistemas; Licenciado en Administración de Empresas; Técnico Superior Universitario en Informática; Técnico Superior Universitario en Administración Mención Mercadotecnia. Componente Docente de la Universidad de los Andes (ULA); Diplomado en Componente Docente en Educación a Distancia; Diplomado Internacional en TIC; Investigador PEII ONCTI - PEII ULA; Experto de la Fundación para la Actualización Tecnológica de Latinoamérica (FATLA) en: Educación Virtual, Administración Web y Comercio Electrónico; dominio de ingles técnico; manejo de programas bajo ambiente Windows; instructor Linux y Web Master.

El contenido de este manuscrito se difunde bajo una Licencia de Creative Commons ReconocimientoNoComercial-Compartirlgual 4.0 Internacional 